\title{
Effect of preemptive analgesia with ibuprofen in the control of postoperative pain in dental implant surgeries: A randomized, triple-blind controlled clinical trial
}

\author{
Gustavo-Mattos Pereira, Luís-Otávio-Miranda Cota, Rafael-Paschoal-Esteves Lima, Fernando-Oliveira Costa
}

Department of Dental Clinics, Oral Surgery and Oral Pathology, School of Dentistry, Federal University of Minas Gerais, Belo Horizonte, Minas Gerais, Brazil

Correspondence:

Department of Periodontology

Federal University of Minas Gerais

Antônio Carlos Avenue, 6627, Pampulha

PO Box 359 Zip Code 31270-901

Belo Horizonte, $M G$ - Brazil

focperio@uol.com.br

Pereira GM, Cota LOM, Lima RPE, Costa FO. Effect of preemptive analgesia with ibuprofen in the control of postoperative pain in dental implant surgeries: A randomized, triple-blind controlled clinical trial. J Clin Exp Dent. 2020;12(1):e71-8.

Received: 07/08/2019 Accepted: 09/12/2019

http://www.medicinaoral.com/odo/volumenes/v12i1/jcedv12ilp71.pdf

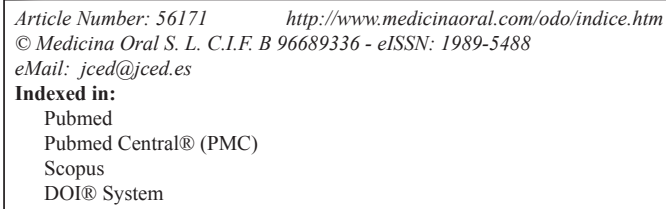

\begin{abstract}
Background: Preemptive analgesia has as its basic principle the administration of analgesics before the onset of painful stimuli, in order to reduce or prevent postoperative pain, but this question is little explored in implantology. Thus, this study was conducted in order to evaluate the clinical efficacy of ibuprofen in pain prevention after unit implant surgery.

Material and Methods: For this triple-blind, parallel, placebo-controlled and randomized clinical trial, 54 insertion surgeries of unitary implants were performed. Two groups have received two different protocols 1 hour before surgery: Ibuprofen group (IBU) $600 \mathrm{mg}$ of ibuprofen; and (2) placebo group (maize starch). The intensity of the pain was evaluated through the visual analogue scale (VAS) in 6 times (1, 6, 12, 24, 48 and 72 hours after the surgery). Patients were instructed to take $750 \mathrm{mg}$ of paracetamol as rescue medication, if necessary. The occurrence and the intensity of pain were analyzed by means of an analysis of variance ANOVA with repeated measurements using the general linear model procedure.

Results: The IBU group had significantly lower VAS scores overall (IBU $=0.30, \pm 0.57$; placebo $=1.14, \pm 1.07$; $p<0.001)$ and at all times in the intra, intergroup comparisons and time/group interaction than the placebo group $(p<0.001)$. The use of rescue medication was significantly lower and the postoperative time was longer in the IBU group compared to placebo $(p=0.002)$.

Conclusions: The single use of ibuprofen was found to be significantly superior in reducing pain after unit implant surgery compared to placebo.
\end{abstract}

Key words: Analgesia, dental implants, pain, surgery, oral. 


\section{Introduction}

Pain is a factor hardly dissociated from dental treatment (1). Their presence or only the possibility of feeling it can trigger innumerable reactions such as fear and anxiety, which can directly interfere with the safety of looking for a particular treatment (2-4). In addition, the dental procedures that generate the greatest fear associated with the possibility of pain include surgical procedures, particularly those of implantology (1).

The concept of preemptive analgesia (PA) to reduce the postoperative pain was based on a series of experimental animal studies (5), which demonstrated central nervous system plasticity and sensitization after nociception. The PA has as basic principle the administration of analgesics before the onset of painful stimuli, in order to reduce or prevent postoperative pain (hyperalgesia), as well as to reduce the analgesic dose required in the postoperative period when compared to the dose used alone, after the occurrence of the pain stimulus (1).

Three classes of analgesic drugs [blockages with local anesthetics, non-steroidal anti-inflammatory drugs (NSAIDs) and opioids] have been used for this purpose alone or in combination $(5,6)$.

Several studies have been conducted in PA, particularly in third molar extraction surgeries (7-12,) periodontal surgeries (13-15), and few studies in implant dentistry $(1,16-18)$, with conflicting results. In addition, different types of drugs have been tested in PA $(1,9,13-16)$ however without a consensual definition of the best protocol regarding the drug, posology and period of exposure to the drug.

Studies have shown a high preference for the use of Ibuprofen in pain control related to dental problems or dental procedures $(12,16,19)$ Ibuprofen, a name derived from the initials of isobutylphenyl propionic acid, is a drug in the group of NSAIDs which acts by non-selectively inhibiting cyclooxygenase 1 and 2 thus avoiding the consequent formation of pro-inflammatory mediators by the arachidonic acid cascade (19).

In this sense, the hypothesis for this study was that individuals who receive PA with the use of ibuprofen (600 mg, oral) 1 hour before the implant surgery present lower postoperative pain in relation to individuals receiving placebo medication.

Thus, the objective of this study was to evaluate and to compare by means of a randomized clinical trial, parallel-controlled and triple blind the efficacy of preemptive administration of Ibuprofen (600 mg oral) 1 hour before surgery for single insertion of implants osseointegrated in the control of postoperative pain.

\section{Material and Methods}

-Study sample

This study was conducted from November 2018 to May 2019 , in a private clinic of Implantology in the city of
Belo Horizonte, Brazil. Fifty-four eligible individuals, both genders, non-smokers, aged 37 to 74 years were selected to participate in this randomized clinical trial, controlled, parallel and triple blind.

The nature of the study was previously explained to each patient, who signed a consent form, and this study was approved by the Ethics Committee in Human Research of the Federal University of Minas Gerais, Brazil (83534618.5.0000.5149) and registered in Brazilian registry of clinical trials (ReBEC) under identifier RBR4B5DSG (www.ensaiosclinicos.gov.br).

The following inclusion criteria were adopted: 1) good systemic health status (ASA I or II); 2) absence of current pain or presence of any oral inflammatory process; 3 ) without analgesics in the 3 weeks prior to the study; 4) lack of continuous use of steroid and non-steroidal anti-inflammatory drugs; 5) need to install unitary implants not concomitant with maxillary and mandibular exodontia; 6) implants of $3.75 \mathrm{~mm}$ in diameter with 11 to $13 \mathrm{~mm}$ in length; 7) presence of compatible bone width and height determined by tomographic examination (cone-bean computed tomography). The following were excluded: pregnant or lactating women, use of some type of medication that could affect the perception of pain, history of allergy or intolerance to the drugs (ibuprofen and paracetamol) used in the research; and history of alcohol or drug abuse.

-Sample Calculation

Using statistical power analysis software R (software, R Foundation, Vienna, Austria), it was determined that 25 patients per group would be required to achieve $80 \%$ of power at $95 \%$ of confidence interval (CI), assuming moderate to intense changes in pain score 24 hours after surgery in at least one group and non-normal distribution data (16). In order to compensate for possible data loss during the study, the number of individuals allocated per group was increased by $10 \%$, with 27 patients per group. Randomization

All patients involved in this study were selected through a sequential non-probability method, that is, consecutive cases were drawn for each eligible intervention using, alternatively, envelopes denominated group I and II in consultation prior to surgery. Randomization in the groups was performed by a sequential stratified randomization process that consisted of the use of 54 opaque envelopes, where the identifications of the treatment groups (groups I and II) were placed. The envelopes were sealed and scrambled, and later numbered in sequential order. For each new entrant in the study, a subsequent numbering envelope was opened. The flow diagram is show in figure 1.

-Medications used

The following drugs were used in this research: Ibuprofen(Alivium ${ }^{\circledR}$ Mantecorp Industry, São Paulo, Brazil) - tablets $600 \mathrm{mg}$, a placebo containing maize starch and 


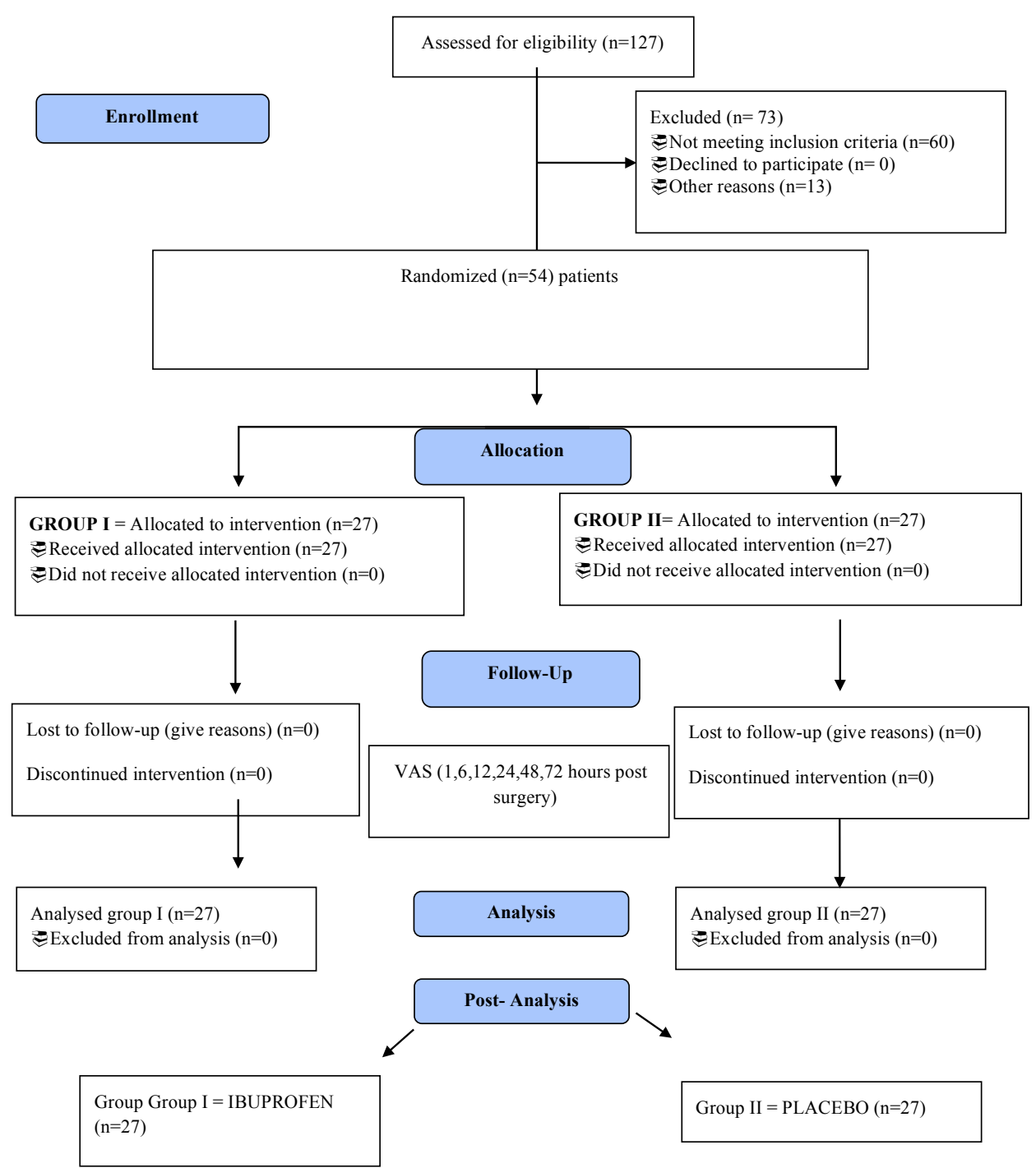

Fig. 1: Flow Diagram.

Paracetamol(Tylenol ® - Johnson \& Johnson, São José dos Campos, Brazil) - tablets $750 \mathrm{mg}$. All drugs used in the research were conditioned in white capsules, placed in bottles identified as group I or II medication by the researcher (G.M.P).

-Instructions for the study and collection of variables All participants in consultation prior to surgery have received detailed instructions for the correct form filling and visual analogue scale (VAS) for postoperative pain assessment. They were advised to contact the operator in case of any doubt. Thus, following randomization procedures, the individuals received a vial containing a medicine capsule from Group I or a medicine capsule from Group II, which should be ingested 1 hour before the surgical procedure. In addition, Paracetamol $750 \mathrm{mg}$ tablets were made available to all individuals to be used as a rescue medication in case of pain. The use of rescue medication was at the discretion of the patient in case of pain. It is noteworthy that all patients registered in this consultation on mobile phones a reminder to take the drug 60 minutes before the scheduled time for surgery. The following information was collected in this consultation: age, gender, body mass index, educational level, family income expressed in minimum salaries, as well as additional information on health in general.

-Visual Analog Scale (VAS)

Participants have completed a visual analogue scale (VAS), where they indicated the presence and intensity of pain $(20,21)$. This scale has $10 \mathrm{~cm}$ of lenght, subdivided into five equal parts, where one end corresponds to no pain (0), and the other end to severe pain $(10,22)$. The registry was completed by all participants at the following times: 1, 6, 12, 24, 48 and 72 hours post-surgery. In addition, in case of the necessity of the use of rescue 
medication, the registration was made at the time of taking the medication in relation to the time elapsed after the end of the surgery.

-Surgical Procedures

Surgical procedures were performed by a single operator (F.O.C.) through a mucoperiosteal flap where unitary implants (Titamax, external hexagon, Neodent, Paraná, Brazil) were inserted according to the manufacturer's instruction. The individuals were anesthetized with a local infiltrative anesthetic (Prilocaine hydrochloride 30 $\mathrm{mg} / \mathrm{ml}$ ) block using 2 to 4 anesthetic tubes at most. All surgical procedures were performed in up to 1 hour.

-Statistical analysis

The analyzes were performed blindly by a researcher (LOMC) continuing to assign the groups as groups I and II. The variables of interest were reported for descriptive measures (mean, standard deviation and $\mathrm{CI}$ ) and analyzed using the chi-square test and t-test when appropriate. The perception of pain over time (VAS 1 hour to 72 hours) between the groups (Placebo vs IBU) was analyzed separately by the Friedmam test (analysis of variance by ranks) and subsequently by means of a 2-way analysis of variance ANOVA with measures repeated through general linear model procedure (GLM).
Comparative analysis of the need for medication rescue for pain control and survival analysis for need of rescue were performed using the Manny-Whitney and Mantel-Cox tests, respectivaly. The data were analyzed using the statistical program (R software). Subsequently the analysis according to the initial knowledgeable investigator (G.M.P.) of the groups has revealed that group I used Ibuprofen (group IBU) and group II the capsule with maize starch (placebo group).

\section{Results}

The study has involved 54 non-smoking patients of both genders, with no significant differences in BMI, surgery time, anatomical position of the implants, socioeconomic and cultural level among the IBU and placebo groups (Table 1). However, they have presented significant differences for age and gender, the IBU group consisted of 12 women and 15 men and the placebo group of 21 women and 6 men $(p=0.012)$. The mean age in the IBU group was $61.07 \pm 8.01$, while in the control group $55.63 \pm 9.36(p=0.026)$ (Table 1). No major adverse effects (infections, major edemas or bleeding) and no side effects of the drugs were reported in both groups.

Table 1: Variables of interest*.

\begin{tabular}{|c|c|c|c|}
\hline Variables & IBU $(n=27)$ & PLACEBO $(n=27)$ & $\boldsymbol{P}$ \\
\hline Age* & $61.07 \pm 8.01$ & $55.63 \pm 9.36$ & 0.026 \\
\hline $\begin{array}{l}\text { Sex } \\
\text { Male } \\
\text { Female }\end{array}$ & $\begin{array}{l}15(55.6 \%) \\
12(44.4 \%)\end{array}$ & $\begin{array}{c}6(22.2 \%) \\
21(77.8 \%)\end{array}$ & 0.012 \\
\hline $\begin{array}{l}\text { Familyincome } * *(\text { BMS })^{\dagger} \\
<5 \text { BMS } \\
\geq 5 \text { BMS }\end{array}$ & $\begin{array}{l}11(40.7 \%) \\
16(59.3 \%)\end{array}$ & $\begin{array}{l}17(62.9 \%) \\
10(47.1 \%)\end{array}$ & 0.086 \\
\hline $\begin{array}{l}\text { Educational level } \\
<8 \text { years } \\
\geq 8 \text { years } \\
\end{array}$ & $\begin{array}{l}13(48.1 \%) \\
14(51.9 \%)\end{array}$ & $\begin{array}{l}12(44.5 \%) \\
15(55.5 \%)\end{array}$ & 0.396 \\
\hline $\begin{array}{l}\text { Body Mass Index } \\
\leq 25 \mathrm{~kg} / \mathrm{m}^{2} \\
>25 \mathrm{~kg} / \mathrm{m}^{2}\end{array}$ & $\begin{array}{c}8(29.6 \%) \\
19(70.4 \%)\end{array}$ & $\begin{array}{c}7(26.0 \%) \\
20(74.0 \%)\end{array}$ & 0.387 \\
\hline $\begin{array}{l}\text { Implant position } \\
\text { Maxilla } \\
\text { Mandible } \\
\end{array}$ & $\begin{array}{l}14(51.9 \%) \\
13(48.1 \%) \\
\end{array}$ & $\begin{array}{l}13(48.1 \%) \\
14(51.9 \%) \\
\end{array}$ & 0.785 \\
\hline $\begin{array}{l}\text { Implant position }{ }^{* *} \\
\text { Anterior } \\
\text { Posterior }\end{array}$ & $\begin{array}{l}15(55.6 \%) \\
12(44.4 \%)\end{array}$ & $\begin{array}{l}10(37.0 \%) \\
17(63.0 \%)\end{array}$ & 0.172 \\
\hline Time of surgery (minutes) * & $49 \pm 5.1$ & $51 \pm 4.2$ & 0.328 \\
\hline
\end{tabular}

Teste t-Student (mean, standart desviation); **Chi-square test (n; \%), †BMS= Brazilian Minimum salary, monthly ( $\sim 300$ american dollars). 
Table 2 and figure 2 shows the comparisons of the scores obtained by the VAS for pain according to the groups and times. Overall, the IBU group (VAS $=0.30, \pm 0.57$ ) had significantly lower VAS scores than the placebo group (VAS
$=1.14, \pm 1.07)(p<0.001)$. In addition, the IBU group also presented values significantly lower than those reported in the control group at all times in the intragroup, intergroup comparisons and in time and group interaction $(p<0.001)$.

Table 2: Comparison of the VAS variable for pain according to groups and times.

\begin{tabular}{|c|c|c|c|c|c|c|c|}
\hline $\begin{array}{l}\text { Group IBU } \\
\mathbf{N}=\mathbf{2 7}\end{array}$ & $\begin{array}{c}\text { VAS } \\
\text { GLOBAL }\end{array}$ & VAS 1H & VAS 6H & VAS 12H & VAS 24H & VAS $48 H$ & VAS 72H \\
\hline Mean & 0.30 & 0.15 & 0.41 & 0.44 & 0.44 & 0.19 & 0.16 \\
\hline s.d. & \pm 0.57 & \pm 0.36 & \pm 0.57 & \pm 0.57 & \pm 0.69 & \pm 0.80 & \pm 0.47 \\
\hline \multicolumn{8}{|l|}{$\begin{array}{l}\text { Group Placebo } \\
\mathbf{N}=\mathbf{2 7}\end{array}$} \\
\hline Mean & 1.14 & 0.74 & 1.56 & 2.22 & 1.56 & 0.48 & 0.26 \\
\hline s.d. & \pm 1.07 & \pm 1.05 & \pm 1.36 & \pm 1.34 & \pm 1.12 & \pm 0.84 & \pm 0.71 \\
\hline $\begin{array}{l}\text { Comparation } \\
\text { intergroups* }\end{array}$ & $<0.001$ & 0.011 & $<0.001$ & $<0.001$ & $<0.001$ & 0.050 & 0.734 \\
\hline Comparation Groups $^{* *}$ & & & & & & & $<0.001$ \\
\hline Comparation Times $^{* *}$ & & & & & & & $<0.001$ \\
\hline Group vs time** & & & & & & & $<0.001$ \\
\hline
\end{tabular}

Friedman test (two-way analysis of variance by ranks)*; ANOVA; general linear model (GLM) **; s.d.=standart desviation.

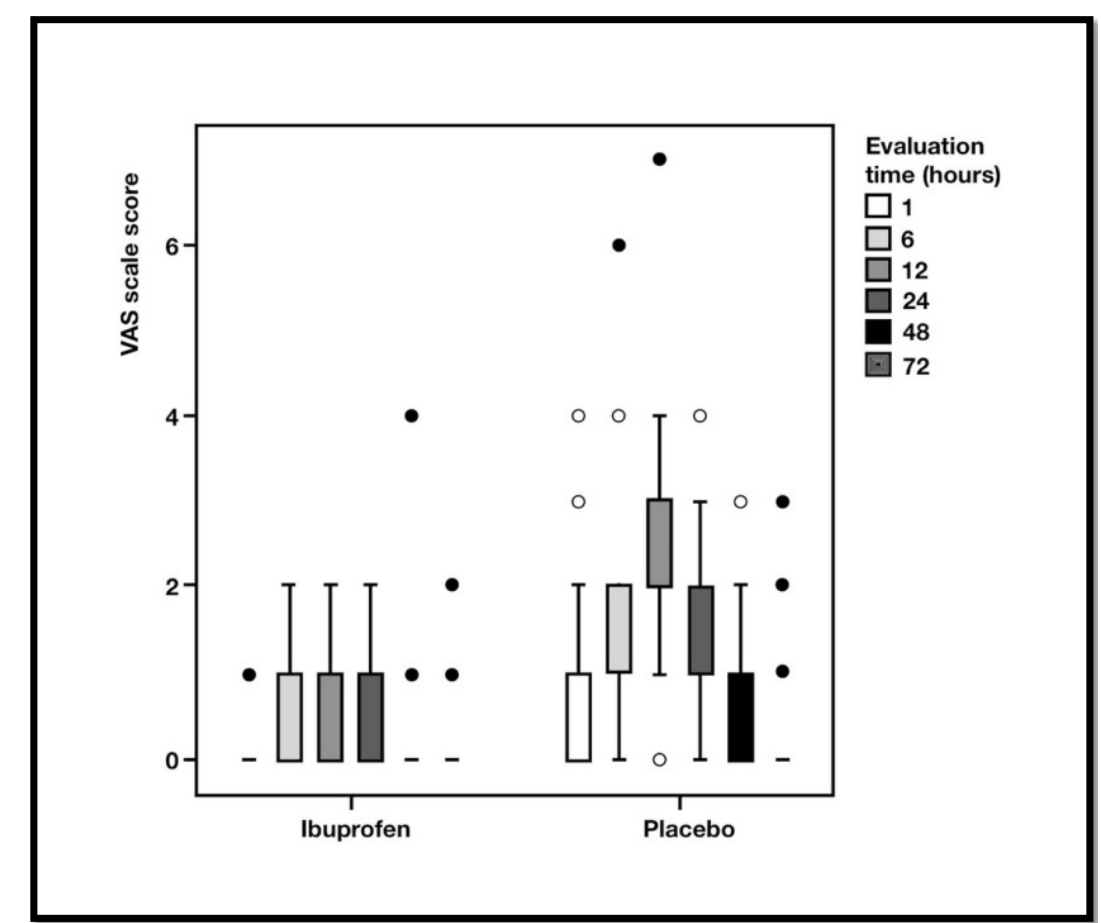

Fig. 2: Boxplot distribuition VAS scores in time by groups. 
Regarding the need to use of the rescue medication to control postoperative pain, the IBU group has used significantly less rescue than that observed in the placebo group (Table 3; Placebo $>$ IBU; $p=0.002$ ).

Corroborating these findings, Table 4 and Figure 3 pre-

Table 3: Comparative analysis of the need for medication rescue for pain control.

\begin{tabular}{|l|c|}
\hline \multicolumn{2}{|l|}{ Group IBU } \\
\hline Mean & 0.81 \\
\hline s.d. & \pm 1.27 \\
\hline Group Placebo & 1.59 \\
\hline Mean & \pm 1.11 \\
\hline s.d. & 0.002 \\
\hline Intergroup comparation & Placebo $>$ IBU \\
\hline
\end{tabular}

Mann-Witney Test ; s.d.=standart desviation. sent a survival analysis of the need to use of the rescue medication, i.e., time elapsed from the first rescue event (mean in hours). In the placebo group the elapsed time was significantly lower (18.85 \pm 4.37 hours) in relation to the IBU group $(45.24, \pm 6.51$ hours, $p=0.007)$.

\section{Discussion}

In this study, the use of ibuprofen 1 hour prior to the surgical procedures for insertion of unitary implants has revealed, overall, a significant effect on reduction of postoperative pain, at all times evaluated, less need for rescue medication, as well as a longer time for the occurrence of the rescue event in relation to the individuals with the use of placebo medication. Thus our hypothesis of its beneficial effect on the reduction of postoperative pain was affirmative.

Theoretically, PA is defined as an antinociceptive treatment (5), which aims to prevent central and peripheral sensitization, reducing or preventing the amplification of postoperative pain. It is believed that this strategy gua-

Table 4: Survival analysis for need of rescue.

\begin{tabular}{|l|c|c|c|c|}
\hline \multirow{2}{*}{ Group } & \multicolumn{3}{|c|}{ Mean } \\
\cline { 2 - 5 } & \multirow{2}{*}{$\begin{array}{c}\text { Estimate } \\
\text { (hours) }\end{array}$} & \multirow{2}{*}{ s.d. } & \multicolumn{2}{|c|}{$95 \%$ CI } \\
\cline { 4 - 5 } & & & Inferior limit & Upper limit \\
\hline IBU & 45.24 & \pm 6.51 & 32.47 & 58.01 \\
\hline Placebo & 18.85 & \pm 4.37 & 10.28 & 27.42 \\
\hline $\boldsymbol{p}$ & & & & 0.007 \\
\hline
\end{tabular}

Equality test (Mantel-Cox).

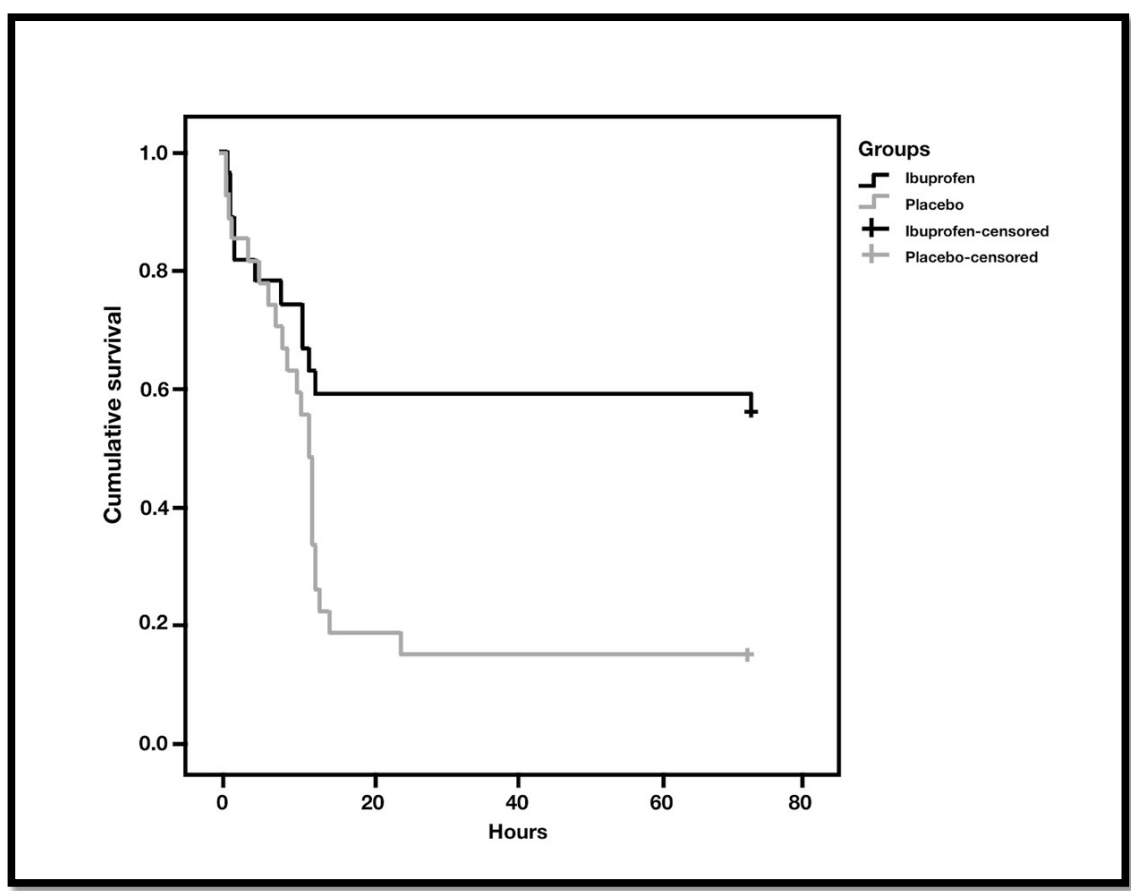

Fig. 3: Survival analysis for need of rescue. 
rantees a reduction in the consumption of analgesics in the postoperative period, providing comfort and reducing the recovery time of the patient $(5,23)$.

In our study, the IBU and placebo groups differed significantly in age and gender, without differences in relation to the other variables studied, such as anatomical position of the implants, surgery time, BMI, family income and socioeconomic level.

Analyzing the plurality of factors that may interfere with perceived pain (4), the absence of differences in almost all variables surveyed between the groups in our study seems to contribute to the validation of our findings. It is known that the level of pain correlated with physical stimuli as a result of dental interventions is difficult to determine, since perceived pain depends on an individual threshold and is influenced by emotional, cognitive and cultural factors (24-27).

In a review about orofacial pain (25) the data were not conclusive about the role of gender and age influencing pain. It has been reported that women are more willing to report pain than men, and that this perception appears to be associated at the pain threshold with respect to heat tolerance. However, after controlling the willingness to report pain, the difference in the thermal pain threshold was no longer statistically significant. In addition, a study on predictors of pain in dental treatment has reported no differences in pain reported in relation to gender and age (24).

Future research needs to elucidate the influence of socio cultural, environmental, and psychological factors on pain as well as the effects of sex on factors that protect against the development of pain or that prevent pain from becoming debilitating (28). Thus, we consider that due to the randomization process and the conflicting pain data regarding gender and age, we do not believe in the interference of this variable in our results.

Several studies have been conducted in PA in dentistry. Most studies focus on third molar extraction surgeries $(7-10,12,28)$ followed by periodontal surgeries $(13-15)$ and studies on implantology (1,16-18).

Moreover, in almost all different procedures using PA, the results are conflicting. In addition, studies with different types of drugs have been tested in PA but without a consensual definition of the best protocol for the drug, posology and period of exposure to the drug. Thus, there is a tendency in PA to test steroid and non-steroidal anti-inflammatory drugs with only one study testing the use of ibuprofen.

In a systematic review (29) it was verified that several clinical trials were conducted seeking to prove the efficacy of PA in the clinical dental practice, however, they exhibited controversial results. According to Kissin (3) it is not possible to detect a preemptive effect in comparative studies of groups in which the analgesic is administered only in the pre-incisional and post-incisional period, due to the complexity in the central sensitization mechanisms and the technical difficulties for the studies, considering it necessary the pre, trans and postoperative analgesic regimen. However, exploring its effects at different time intervals may bring further clarification of its effectiveness. In this context, this study has searched for this information in periods ranging from 1 to 72 hours, as well as the additional need for rescue medication for pain control.

Additionally, in our findings, ibuprofen has proven to be very effective in reducing pain in the immediate postoperative period from 1 to 6 hours compared to placebo, significantly reducing the need for rescue medication and increasing the time required for rescue medication, thus according to the theoretical foundations of PA this initial reduction is very important because it has a positive effect on reducing pain at subsequent intervals of time $(1,5,23)$.

In this context, our finding is supported by a systematic review (29) of the oral kinetics of oral ibuprofen (30 studies evaluating 1,015 individuals) which showed that mean maximum plasma concentrations of fast acting formulations occurred within 50 minutes (29-35 minutes for arginine, lysine and sodium salts) compared to 90 minutes for standard formulations. Initial rapid reduction of pain intensity was also associated with reduced need for remedies. Additionally, the ibuprofen by inhibiting the production of prostaglandins, it leaves the gastric mucosa less protected against acidity, being its therapeutic efficacy easily seen overcoming the severity of its side effects (19).

Another point to be discussed refers to the extent and numbers of implants inserted in the surgical procedure. Low pain scores are related to implant surgeries and according to Kim et al. (27) pain and anxiety scores are proportional to the number of implants inserted and the length of the surgical procedure. However, regarding these questions, our study presents the advantages of standardization of insertion of only one implant, with a single operator and similar surgery time between the groups.

The present study may be considered as a good starting point for future studies aimed at providing additional information on the use of ibuprofen in PA in implant surgeries. However, prospective multicentric studies, in different populations, with different surgical and dosing protocols, are necessary to confirm these findings and to establish a beneficial protocol for the control of pain in dental implant surgeries.

The present study demonstrated that the use of ibuprofen 1 hour before to the surgical procedures for insertion of unitary implants has revealed a significant effect on the reduction of postoperative pain overall at all evaluated times has shown less need for the use of rescue medication and, when necessary, the time of use has been 
shorter when compared to individuals on placebo. Thus, its use can be considered a beneficial adjuvant for the control of postoperative pain in dental implant surgeries.

\section{References}

1. Sánches-Pérez A, Muñoz-Peñalver $\mathrm{J}$, Moya-Villaescusa $\mathrm{Mj}$, Sánches-Matás C. Effects of the preoperative administration of dexketoprofen trometamol on pain and swelling after implant surgery: A randomized, double-blind controlled trial. J Oral Implantol. 2018;44:122-129.

2. Thomson PI, Rood JP. Mental nerve dysfunction: a symptom of diverse mandibular disease. Dent Update. 1995;22:271-274.

3. Kissin I. Preemptive analgesia. Anesthesiol. 2000;93:1138-1143.

4. Santuchi CC, Cortelli SC, Cortelli JR, Cota LO, Alencar CO, Costa FO. Pre- and post-treatment experiences of fear, anxiety, and pain among chronic periodontitis patients treated by scaling and root planing per quadrant versus one-stage full-mouth disinfection: a 6-month randomized controlled clinical trial. J Clin Periodontol. 2015;42:102431.

5. Kelly DJ, Ahmad M, Brull SJ. Preemptive analgesia I: physiological pathways and pharmacological modalities. Can J Anaesth. 2001;48:1000-1010.

6 . Hite, PF. The changing role of non-opioid analgesic techniques in the management of postoperative pain. Anesth Analg. 2005;101:S522.

7. Alcântara CEP, Falci SGM, Oliveira-Ferreira F, Santos CRR, Pinheiro MLP. Pre-emptive effect of dexamethasone and methylprednisolone on pain, swelling, and trismus after third molar surgery: A split-mouth randomized triple-blind clinical trial. Int J Oral Maxillofac Surg. 2014;43:93-98.

8. Araújo FAC, Santos TS, Morais HHA, Silva EDO, Vasconcelos RJH. Comparative analysis of preemptive analgesic effect of tramadol chlorhydrate and nimesulide following third molar surgery. J Craniomaxillofac Surg. 2012;40:e346-e349.

9. Gazal G, Al-Samadani KH. Comparison of paracetamol, ibuprofen, and diclofenac potassium for pain relief following dental extractions and deep cavity preparations. Saudi Med J. 2017;38:284-291.

10. Demirbas AE, Karakaya M, Bilge S, Canpolat DG, Kütük N, Alkan A. Does single-dose preemptive intravenous ibuprofen reduce postoperative pain after third molar surgery? A prospective, randomized, double-blind clinical study. J Oral Maxillofac Surg. 2019;77:19901997.

11. Simone JL, Jorge WA, Horliana ACRT, Canaval TG, Tortamano I P. Comparative analysis of preemptive analgesic effect of dexamethasone and diclofenac following third molar surgery. Braz Oral Res. 2013;27:266-271.

12. Weiser T, Richter E, Hegewisch A, Muse DD, Lange R. Efficacy and safety of a fixed-dose combination of ibuprofen and caffeine in the management of moderate to severe dental pain after third molar extraction. Eur J Pain. 2018;22:28-38.

13. Pilatti GL, André SF, Bianchi A, Cavassin R, Tozetto CW. The use of celecoxib and dexamethasone for the prevention and control of postoperative pain after periodontal surgery. J Periodontol. 2006;77:1809-1814.

14. Steffens JP, Santos FA, Sartori R, Pilatti Gl. Preemptive dexamethasone and etoricoxib for pain and discomfort prevention after periodontal surgery: a double-masked, crossover, controlled clinical trial. J Periodontol. 2010;81:1153-1160.

15. Steffens JP, Santos FA, Pilatti Gl. The use of etoricoxib and celecoxib for pain prevention after periodontal surgery: a double-masked, parallel-group, placebo-controlled, randomized clinical trial. J Periodontol. 2011;82:1238-1244.

16. Ahammam MA, Kayal RA, Alasmari DS, et al. Comparison between dexamethasone and ibuprofen for postoperative pain prevention and control after surgical implant placement: a double-masked, parallelo-group, placebo-controlled randomized clinical trial. J Periodontol. 2017;88:69-77.
17. Meta IF, Bermolen M, Macchi R, Aguilar J. Randomized controlled trial comparing the effects of 2 analgesic drug protocols in patients who received 5 dental implants. Implant Dent. 2017;26:412-416.

18. Samieirad S, Afrasiabi H, Tohidi E, et al. Evaluation of caffeine versus codeine for pain and swelling management after implant surgeries: A triple blind clinical trial. J Craniomaxillofac Surg. 2017;45:1614-1621.

19. Irvine J, Afrose A, Islam N. Formulation and delivery strategies of ibuprofen: challenges and opportunities. Drug Dev Ind Pharm. 2018;44:173-83.

20. Moore RA, Derry S, Straube S, et al. Faster, higher, stronger? Evidence for formulation and efficacy for ibuprofen in acute pain. Pain. 2014;155:14-21.

21. Huskisson E. Measurement of pain. The Lancet. 1974;304:11271131.

22. Pasqualini D, Cocero A, Castella L, Mela L, Bracco P. Primary and secondary closure of the surgical wound after removal of impacted mandibular third molars: a comparative study. Int J Oral Maxillofac Surg. 2005;34:52-57.

23. Ong CKS, Lirk P, Seymour RA, Jenkins BJ. The efficacy of preemptive analgesia for acute postoperative pain management: A meta-analysis. Anesth Analg. 2005;100:757-773.

24. Tickle M, Milsom K, Crawford FI, Aggarwal VR. Predictors of pain associated with routine procedures performed in general dental practice. Community Dent Oral Epidemiol. 2012;40:343-350.

25. Shaefer JR, Khawaja SN, Bavia PF. Sex, gender, and orofacial pain. Dent Clin North Am. 2018;62:665-682.

26. Armfield JM, Spencer AI, Stewart JF. Dental fear in Australia: who's afraid of the dentist? Aust Dent J. 2006;51:78-85.

27. Kim S, Lee VJ, Lee S, Moon HS, Chung MK. Assessment of pain and anxiety following surgical placement of dental implants. Int J Oral Maxillofac. Implants. 2013;28:531-535.

28. Simone JL, Jorge WA, Horliana AC, Canaval TG, Tortamano IG. Comparative analysis of preemptive analgesic effect of dexamethasone and diclofenac following third molar surgery. Braz Oral Res. 2013;27:266-271.

29. Collins S, Moore RA, Mcquay HJ, et al. Single dose oral ibuprofen for acute postoperative pain in adults. Cochrane Database Sys Rev. 2015;1:1-68.

\section{Conflict of interest}

The authors have declared that no conflict of interest exist. 